OPEN ACCESS

Edited by:

Athanasios Papatsoris,

Sismanogleio General

Hospital, Greece

Reviewed by:

Nikos Kostakopoulos, Aberdeen Royal Infirmary,

United Kingdom

Philipp Mandel,

University Hospital Frankfurt, Germany

*Correspondence:

Markus Angerer

markusangererhh@gmail.com

Specialty section: This article was submitted to

Genitourinary Surgery,

a section of the journal

Frontiers in Surgery

Received: 23 October 2020 Accepted: 08 December 2020

Published: 03 February 2021

Citation:

Angerer $M$, Salomon $G$ BeyersdorffD, Fisch M, Graefen M and Rosenbaum CM (2021) Impact of

Sarcopenia on Functional and Oncological Outcomes After Radical Prostatectomy.

Front. Surg. 7:620714

doi: 10.3389/fsurg.2020.620714

\section{Impact of Sarcopenia on Functional and Oncological Outcomes After Radical Prostatectomy}

\author{
Markus Angerer ${ }^{1,2 \star}$, Georg Salomon ${ }^{1}$, Dirk Beyersdorff ${ }^{3}$, Margit Fisch ${ }^{2}$, Markus Graefen ${ }^{1}$ \\ and Clemens M. Rosenbaum ${ }^{4}$
}

${ }^{1}$ Martini-Klinik Prostate Cancer Center, University Hospital Hamburg-Eppendorf, Hamburg, Germany, ${ }^{2}$ Department of Urology, University Hospital Hamburg-Eppendorf, Hamburg, Germany, ${ }^{3}$ Department of Radiology, Interventional Radiology and Nuclear Medicine, University Hospital Hamburg-Eppendorf, Hamburg, Germany, ${ }^{4}$ Department of Urology, Asklepios Hospital Barmbek, Hamburg, Germany

Introduction and Objectives: Knowledge about the significance of sarcopenia (muscle loss) in prostate cancer (PCa) patients is limited. The aim of this study was to determine the influence of skeletal muscle index (SMI) on early functional and pathological outcome in patients undergoing radical prostatectomy (RP).

Materials and Methods: One hundred randomly chosen patients who received RP between November 2016 and April 2017 at Martini-Klinik (Hamburg, Germany) were retrospectively assessed. SMI (skeletal muscle mass cross-sectional area at $\mathrm{L} 3 / \mathrm{m}^{2}$ ) was measured by preoperative staging computed tomography scans at L3 level. Cox regression analysis was applied to determine the impact of SMI on post-operative outcome. Follow-up was 12 months. Continence was defined as no more than one safety pad per day.

Results: Mean age of the cohort was 63.6 years. Mean SMI was $54.06 \mathrm{~cm}^{2} / \mathrm{m}^{2}$ (range, $40.65-74.58 \mathrm{~cm}^{2} / \mathrm{m}^{2}$ ). Of the patients, $41.4 \%$ had $\mathrm{pT} 2,28.7 \%$ had pT3a, and $29.9 \%$ had pT3b or pT4 PCa. SMI revealed to be without significant correlation on tumor stage. Follow-up data of 55 patients were available for early functional outcome analysis. SMI showed no significant influence on erectile function in multivariable Cox regression analysis. In multivariable Cox regression analysis, SMI turned out to have no influence on continence rates 6 weeks after surgery.

Conclusion: The present study shows that patients undergoing RP have a wide range of SMI. Unlike in other urological malignancies, there was no significant impact of SMI on early functional outcome and pathological outcome. A larger cohort is needed to confirm these results.

Keywords: prostate cancer, sarcopenia, radical prostatectomy, oncological outcome, functional outcome

\section{INTRODUCTION}

Prostate cancer $(\mathrm{PCa})$ is the most common cancer and the third most common cause of cancer death among men in the western world (1). According to the German health report in corporation with the Robert-Koch-Institute, $\sim 49,000$ cases of PCa are reported per annum; the incidence is 120 in all age classes in Germany (2). 
Radical prostatectomy (RP), brachytherapy (BT), and the advanced technique of radiation using intensity-modulated radiation therapy (IMRT) are the three most common treatment procedures for localized prostate cancer. All techniques show no significant contrariness in overall survival $(3,4)$. RP embodies one of the most often used treatment option in localized prostate cancer, mainly implemented as either retropubic open RP or laparoscopic/robot-assisted RP (5).

The most recognized risk factors for developing $\mathrm{PCa}$ are increasing age, ethnic origin, and family history (6). The familiar predisposition suggests an inherited genetic component to $\mathrm{PCa}$ $(7,8)$. Preoperative prostate-specific antigen (PSA), pathological stage, Gleason score, and surgical margins status predicted BCR after RP (9).

"Sarcopenia is a progressive and generalized skeletal muscle disorder that is associated with increased likelihood of adverse outcomes including falls, fractures, physical disability, and mortality" as defined by the European Working Group on Sarcopenia in Older People (EWGSOP) (10). Sarcopenia is increasingly recognized as a risk factor for a worse performance especially in patients suffering from a malignant tumor disease (11). Lately, the presence of sarcopenia has been identified as a "prognostic marker of disease recurrence, cancer-specific mortality (CCM), and all-cause mortality (ACM)" in patients with not particularly urological malignancies but also, e.g., gynecological and gastrointestinal cancer diseases (12-15).

The definition of sarcopenia is based on the skeletal muscle index (SMI). The muscle volume can reproducibly be measured by computed tomography (CT) or magnetic resonance imaging (MRI) (10).

Among others, potential risk factors of perioperative complications are BMI $>30$ and Charlson comorbidity index (CCI) $\geq 1$ (16). Performance status and comorbidity are generally subjective and difficult to define. The American Association of Anesthesiologists (ASA) score, the Eastern Cooperative Oncology Group (ECOG) performance status, and CCI are commonly calculated prognostic factors for analyzing postoperative outcomes. Yet, they have been doubted to identify those patients at highest risk of perioperative morbidity and mortality, despite the successfully recognition of all status $(14,17)$. Sarcopenic patients have been demonstrating a higher rate of perioperative complications (18-21).

This resulted to proclaim sarcopenia as an important acknowledging factor in treatment planning, decision-making, and gaining information regarding patients peri- and postoperative outcome (17).

In men diagnosed with prostate cancer, little is known about the role of sarcopenia influencing the functional and oncological outcome. One study concluded that sarcopenia does not predict

\footnotetext{
Abbreviations: $\mathrm{PCa}$, prostate cancer; RP, radical prostatectomy; IMRT, intensity modulated radiotherapy, BT, brachytherapy; EWGSOP, European Working Group on Sarcopenia in Older People; ACM, all-cause mortality; CCM, cancer-specific mortality; MRI, magnetic resonance imaging; CCI, Charlson comorbidity index; ECOG, Eastern Cooperative Oncology Group; ASA, American Association of Anesthesiologists; SMI, skeletal muscle index; OS, overall survival; CT, computed tomography; HU, Hounsfield unit; BMI, body mass index; HR, hazard ratio; CI, confidence interval.
}

the oncological outcome after RP (22). Another study that investigated men undergoing radiotherapy for PCa identified a significant impact of skeletal muscle reduction on non-cancer mortality (23).

We hypothesized that sarcopenia may be correlate with a higher complication rate and worse oncological outcome in men undergoing RP. Consequently, we examined the association between sarcopenia and perioperative as well as oncological outcome in men undergoing RP (17).

\section{MATERIALS AND METHODS}

We retrospectively analyzed 100 patients who were treated with RP, either open retropubic RP or laparoscopic, robot-assisted $\mathrm{RP}$ at a high-volume center (Martini-Klinik Prostate Cancer Center, Hamburg-Eppendorf, Germany) between November 2016 and April 2017. RP was only performed consistently by eight highly trained surgeons performing RRP and robot-assisted $\mathrm{RP}$ regularly.

We have identified the patients randomly within our database. Staging CT scans were obtained by patients with intermediateand high-risk PCa defined by D'Amico as a clinical $\mathrm{T}$ stage $\geq \mathrm{cT} 2 \mathrm{c}$, a Gleason score $\geq 8$, or a PSA $>20 \mathrm{ng} / \mathrm{ml}$ (24).

CT images were obtained from the patients preoperative CT scans of the abdomen or pelvis. Included were only patients with sufficient quality of CT images. Patients' informed consent for data collection was obtained. The cross-sectional area of all skeletal muscle at third lumbar vertebrae 3 (L3) has a high correlation to the body's general muscle volume (18). Lumbal SMI is calculated by the cross-sectional area of all skeletal muscle at L3 by height squared $\left(\mathrm{m}^{2}\right)$ and reported as $\mathrm{cm}^{2} / \mathrm{m}^{2}$. Clinical, blood sample results, and oncological data were collected from the hospitals' documenting program, Soarian, and Martini Data Registry.

A single axial image at the level of L3 was selected, and the cross-sectional area of all skeletal muscle at L3 was measured after identifying the muscle-specific attenuation thresholds $(-29-$ $150 \mathrm{HU}$ ). For the measurement, musculus rectus abdominus; internal, external, and lateral musculus obliquus abdominis; musculus psoas; musculus quadratus lumborum; and musculus erector spinae were included. Axial CT images at L3 vertebra depicting patient without sarcopenia are shown in Figures 1A,B as compared to patients with different BMIs and significantly different SMI shown in Figures 1C,D. The radiologist program Centricty Viewer GE was used for image analysis. Image analysis was performed by the same investigator who was unaware about the patients' cancer-specific data.

Clinical and pathological data were collected. Clinical data are include information on age, clinical TNM classification (clinical tumor and lymph node stage), preoperative PSA, continence by the number of pad usage per day, as well as preoperative androgen deprivation therapy. Pathological data collected included prostate biopsy Gleason score, pathological specimen Gleason score, pathological TN classification (pathological tumor and lymph node stage), and surgical margin status. 


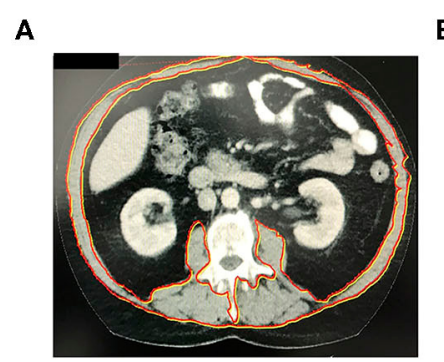

B

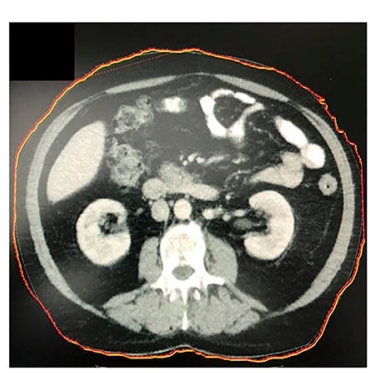

C

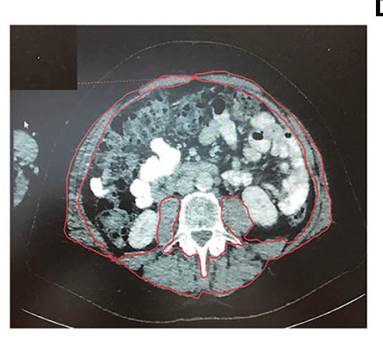

D

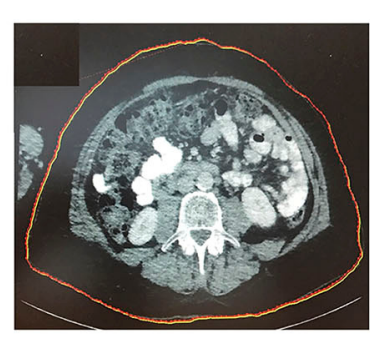

FIGURE 1 | (A,B) Axial CT-image at L3 vertebra depicting patient without sarcopenia. (C,D) Axial CT-image at L3 vertebra depicting patient with BMI and significantly different SMI (sarcopenic patient). The red marked area represents the cross-sectional area of all skeletal muscle at L3 including the rectus abdominus; internal, external, and lateral obliques; psoas; quadratus lumborum; and erector spinae muscles. The red marked line in the image represents patients abdominal circumference.

Taking into consideration the EWGSOP definition of sarcopenia, SMI was based on sex- and BMI-specific cutoffs for men $<43 \mathrm{~cm}^{2} / \mathrm{m}^{2}(\mathrm{BMI}<25)$ and $<53 \mathrm{~cm}^{2} / \mathrm{m}^{2}(\mathrm{BMI}>25)$ to classify patients as sarcopenic vs. non-sarcopenic (25).

Urine continence was defined not to use more than 1 safety Pad per Day.

\section{Statistical Analysis}

Clinical and pathological variables were compared between the sarcopenic and non-sarcopenic patients. Age, BMI (in $\mathrm{kg} / \mathrm{m}^{2}$ ), pathological tumor and lymph node stage, pathological surgical margin status, PSA, and Gleason score are taken into account for comparison of the two groups. Continuous features were summarized with medians and interquartile ranges (IQRs). Categorical features were summarized with frequency counts and percentages and compared using the chi-square test. The primary interest was to evaluate the functional and oncological outcome.

Logistic regression analysis was used to estimate the oncological outcome and biochemical recurrence (BCR). BCR was defined as PSA value $>0.2 \mathrm{ng} / \mathrm{ml}$ after RP. Urine continence was assessed by univariable and multivariable Cox proportional hazards regression models and summarized with hazards ratios (HRs) and 95\% confidence intervals (95\% CIs).

Furthermore, statistically significant prognosticators on univariable analysis were also analyzed in multivariable models. A $p<0.05$ was considered to be statistically significant.

For follow-up assessment, patients were evaluated for urinary continence and erectile function (EF) after 6 weeks and 12 months after RP. Patient-reported outcomes were registered by standardized Martini-Klinik questionnaires (5).

\section{RESULTS}

We included for the first analysis 99 patients from our database who fulfilled the inclusion criteria. All of them were operated between November 2016 and April 2017. One patient was excluded due to missing data.

SMI measurements of all 99 patients were conducted based on SMI definition; 26 patients (26.3\%) were classified as sarcopenic.
Descriptive pathological and perioperative characteristics are shown in Table 1.

Overall, sarcopenic patients were older than non-sarcopenic patients (mean age, 68.0 vs. 64 years, $p=0.02$ ). There was no difference between sarcopenic and non-sarcopenic patients in local and lymphonodal pathologic stage or Gleason score. There was no significant difference between sarcopenic and nonsarcopenic patients regarding nerve-sparring surgery (84.9 vs. $88.5 \%, p=0.91)$.

In addition, there was no significant difference in urine continence at 1 year after surgery between sarcopenic and nonsarcopenic patients in multivariable logistic regression analysis [odd's ratio (OR), 1.05; 95\% confidence interval (CI), 0.96-1.16; $p=0.26]$.

Results are shown in Table 2.

In Cox regression analysis, the incidence of BCR did not differ significantly 1 year after surgery between sarcopenic and nonsarcopenic patients [hazard ratio (HR), 0.97; 95\% CI, 0.3-3.08; $p=0.953]$.

\section{DISCUSSION}

Sarcopenia represents "a response to both nutrient deprivation and systemic stress, resulting in critical anatomic and functional deficits" (17). Sarcopenia is a major public health issue. Using the definition with highest prevalence estimates, the number of individuals with sarcopenia would rise from 19,740 million in 2016 to 32,338 million in 2045 only in Europe, corresponding to an increase from 20.2 to $22.3 \%$ (26).

In this current study, we examined the association between sarcopenia and functional and oncological outcome after RP. Our hypothesis that sarcopenia significantly effects functional and oncologic outcome in men undergoing RP could not be proven.

We noted several findings of interest. First, we determined that in this cohort of patients with RP, $26.3 \%$ of patients were classified as sarcopenic preoperatively. The median age of sarcopenic patients was significantly older.

The correlation between BMI and outcome after RP has been investigated often in past. An increase in BMI 
TABLE 1 | Descriptive pathologic and perioperative characteristics of PCa patients that underwent RP between November 2016 and April 2017.

\begin{tabular}{|c|c|c|c|c|}
\hline Characteristics & Overall & Non-sarcopenic patients & Sarcopenic patients & $p$-value \\
\hline No. of patients, $n(\%)$ & 99 & $73(73.7)$ & $26(26.3)$ & \\
\hline Age at RP (years), median & $65(59-68.7)$ & $64(57-67)$ & $68(61-71)$ & 0.02 \\
\hline Prostate volume (ml), median & $39(30-47.5)$ & $38(28-48)$ & $41(33-46)$ & 0.19 \\
\hline SMI $\left(\mathrm{cm}^{2} / \mathrm{m}^{2}\right)$, median & $54(49.4-58.6)$ & $57(53-61)$ & $50(46-50)$ & $<0.001$ \\
\hline \multicolumn{5}{|l|}{ Nerve-sparing (\%), $n$} \\
\hline Yes & 85 & $62(84.9)$ & $23(88.5)$ & 0.91 \\
\hline No & 14 & $11(15.1)$ & $3(11.5)$ & \\
\hline pT-Stadium (\%), $n$ & & & & 0.81 \\
\hline pT2 & 42 & $32(43.8)$ & $10(38.5)$ & \\
\hline pT3/4 & 57 & $41(56.2)$ & $16(61.5)$ & \\
\hline pN-Status (\%), $n$ & & & & 0.29 \\
\hline $\mathrm{Nx} / \mathrm{NO}$ & 66 & $46(63)$ & $20(67.9)$ & \\
\hline $\mathrm{N}+$ & 33 & $27(37)$ & $6(23.1)$ & \\
\hline Gleason (\%), $n$ & & & & 0.35 \\
\hline $3+3 / 3+4$ & 40 & $27(37)$ & $13(50)$ & \\
\hline $4+4 />4+4$ & 59 & $46(63)$ & $13(50)$ & \\
\hline
\end{tabular}

TABLE 2 | Urine continence at 1 year after RP.

\begin{tabular}{lccc}
\hline Characteristics & Odd's ratio & $\mathbf{9 5 \%} \mathbf{~ C l}$ & $\boldsymbol{p}$-value \\
\hline Age at RP & 1.05 & $0.95-1.17$ & 0.31 \\
Prostate volume & 1.02 & $0.98-1.06$ & 0.4 \\
Nerve-sparing & & & \\
Yes & Reference & & 0.4 \\
No & 0.38 & $0.03-3.35$ & \\
pT-Stadium & & & 0.43 \\
pT2 & Reference & $0.1-2.46$ & 0.42 \\
pT3a & 0.53 & $0.35-10.92$ & 0.26 \\
pT3b/pT4a & 1.99 & $0.96-1.16$ & \\
SMl & 1.05 & & \\
\hline
\end{tabular}

showed a significant increase risk of peri- and post-operative complications; prolonged operative time, increased blood loss, increased open conversions, longer hospitalization, and higher positive surgical margin rate (27). BMI has known associations with diabetes, coronary artery disease, and hypertension (27). Obesity also has a significant impact on mortality in cancer patients (24). Freedland et al. concluded that elevated BMI has been associated with biochemical failure after radical prostatectomy, due to inferior surgery, which caused a higher rate of positive surgical margin. Also in their cohort, obese men after RP showed worse outcomes, suggesting that obesity may be associated with a biologically more aggressive form of prostate cancer (28-30). Still, it remains controversial regarding the effect on BCR (22).

As mentioned before, $\mathrm{McD}$ onald et al. assessed in their study the cross-sectional area at the L4-5 level after radiotherapy for localized prostate cancer retrospective of 653 men (23). They were concluding that sarcopenia significantly increased risk of non-cancer mortality after radiotherapy. Analyzing their cohort, the conclusion is due to the fact that cross-sectional area of all total skeletal muscle was measured at L4-5 and relatively few patients. Furthermore, this study had muscle L4-5 values below the sarcopenic threshold.

Mason et al. published in June 2018 the association between sarcopenia and oncological outcome after RP in a cohort of totally 698 patients and 310 patients identified as sarcopenic (22). They concluded that sarcopenia has no significant association with either perioperative complications or oncological outcome after RP. This study showed a representative number of patients classified sarcopenic (55.6\%). Furthermore, there were no significant differences in clinical $\mathrm{T}$ or $\mathrm{N}$ stage or biopsy Gleason score.

Two different cohort of men with prostate cancer showed contradictory associations of sarcopenia. This may be because of the different populations or different cancer-specific criteria. Patients for RP selected by urologists favoring patients younger in age with a longer life expectancy and reduced comorbidities.

Our data reveal that SMI has neither significant influence on pathological outcome nor on BCR rates after RP.

Furthermore, SMI had no impact on post-operative urine continence in our cohort. These results may suggest that sarcopenia is not a prognostic marker for functional and oncological outcome after RP.

In our study, we acknowledge several limitations to this study. First, we cannot rule out a bias due to random selection of included patients. Not all patients between the period of November 2016 and April 2017 who underwent RP have been selected for analysis and follow-up. Exclusion was caused by missing CT scans, either not readable, poor quality for analysis or missing import; or low-risk PCa patients accordingly to D'Amico classification, which have not received a preoperative CT staging. Another major limitation is that our cohort only figured 99 patients. Therefore, additional subanalyses of risk classifications are necessary. SMI was only measured by preoperative scans. The 
change in SMI is not considered. Ha et al. showed a significant change in sarcopenia and SMI 1 year after radical cystectomy and might be an effective marker for oncological outcome (31). Another limitation of this study is the time of follow-up after $\mathrm{RP}$, which limits the statement of sarcopenia effecting BCR. The results currently show the 12 months questionnaire feedback. The effect of BCR cannot safely be clarified; hence, the followup time must be prolonged. We are continuing to assess followup data.

Nevertheless, little is known about the association of sarcopenia on functional and oncological outcome after RP. Our study presents that sarcopenia is not significantly associated with influencing the oncological outcome, urine continence, or BCR after RP.

\section{CONCLUSION}

Sarcopenia was not significantly associated with worse functional and oncological outcome after RP. In addition, sarcopenia has no significant effect on BCR. Thus, sarcopenia is not a prognostic marker for patients with prostate cancer after RP.

\section{DATA AVAILABILITY STATEMENT}

The raw data supporting the conclusions of this article will be made available by the authors, without undue reservation.

\section{REFERENCES}

1. Siegel RL, Miller KD, Jemal A. Cancer statistics, 2020. CA Cancer J Clin. (2020) 70:7-30. doi: 10.3322/caac. 21590

2. Rohde V, Katalinic A, Wasem J, Aidelsburger P. Gesundheitsberichterstattung des Bundes Heft 36 Prostataerkrankungen. Arnhem: Robert Koch Institut; Statistisches Bundesamt (2007).

3. Hayashi N, Yokomizo Y, Osaka K, Makiyama K, Nakaigawa N, Yao $\mathrm{M}$, et al. Ten year outcomes of treatment for localized prostate cancer in a single institution; comparison of radical prostatectomy vs radiation therapy-prospensity score matching analysis. J Urol. (2016) 195:247784. doi: 10.1016/j.juro.2016.02.2882

4. Mottet N, Bellmunt J, Briers E, Bolla M, Bourke L, Cornford P, et al. EAUESTRO-ESUR-SIOG guidelines on prostate cancer. In: Presented at the EAU Annual Congress Amsterdam 2020. Berlin: EAU Guidelines Office (2020).

5. Haese A, Knipper S, Isbarn H, Heinzer H, Tilki D, Salomon G, et al. A comparative study of robot-assisted and open radical prostatectomy in 10790 men treated by highly trained surgeons for both procedures. BJU Int. (2019) 123:1031-40. doi: 10.1111/bju.14760

6. Key T. Risk factors for prostate cancer. Cancer Surv. (1995) 23:63-77.

7. Lichtenstein P, Holm NV, Verkasalo PK, Iliadou A, Kaprio J, Koskenvuo M. Environmental and heritable factors in the causation of cancer-analyses of cohorts of twins from Sweden, Denmark, and Finland. N Engl J Med. (2000) 343:78-85. doi: 10.1056/NEJM200007133430201

8. Oh SJ, Mandel P, Chun FKH, Tennstedt P, Peine S, Hohenhorst JL, et al. AB0/Rhesus blood group does not influence clinicopathological tumor characteristics or oncological outcome in patients undergoing radical prostatectomy. Front Surg. (2017) 4:75. doi: 10.3389/fsurg.2017. 00075

9. Ouzzane A, Koenig P, Ballereau C, Zini L, Ghoneim T, Maladry F, et al. Oncologic outcomes after radical prostatectomy: French validation of the D'Amico Risk Group classification. Prog Urol. (2010) 20:120612. doi: 10.1016 j.purol.2010.05.015

\section{ETHICS STATEMENT}

The studies involving human participants were reviewed and approved by Ethikkommission Hamburg. The patients/participants provided their written informed consent to participate in this study.

\section{AUTHOR CONTRIBUTIONS}

MA was responsible for conceiving the presented idea, designed the study, developed the theory, and performed the computations, literature research, data collection, writing of the manuscript with the help of CR, CT image analysis, and statistical calculations. GS contributed with literature research, investigated, and supervised the findings of this work, helped with manuscript correction and revision. DB contributed with the help of CT image analysis and providing the software, also helped with manuscript correction and revision. MF contributed with the help of manuscript correction and revision. MG contributed with literature, verified the analytical methods, helped with manuscript correction and revision. CR contributed with planning and supervision of the work, helped with statistical calculations, manuscript correction and revision. All authors discussed the results and contributed to the final manuscript.
10. Cruz-Jentoft AJ, Bahat G, Bauer J, Boirie Y, Bruyere O, Cederholm T, et al. Sarcopenia: revised European consensus on definition and diagnosis. Age Ageing. (2019) 48:16-31. doi: 10.1093/ageing/afz046

11. Joglekar S, Nau PN, Mezhir JJ. The impact of sarcopenia on survival and complications in surgical oncology: a review of the current literature. J Surg Oncol. (2015) 112:503-9. doi: 10.1002/jso.24025

12. Pinsky PF, Kramer BS, Reding D, Buys S. Reported family history of cancer in the prostate, lung, colorectal, and ovarian cancer screening trial. Am J Epidemiol. (2003) 157:792-9. doi: 10.1093/aje/kwg043

13. Del Fabbro E, Parsons H, Warneke CL, Pulivarthi K, Litton JK, Dev R, et al. The relationship between body composition and response to neoadjuvant chemotherapy in women with operable breast cancer. Oncologist. (2012) 17:1240-5. doi: 10.1634/theoncologist.2012-0169

14. Peng P, Hyder O, Firoozmand A, Kneuertz P, Schulick RD, Huang D, et al. Impact of sarcopenia on outcomes following resection of pancreatic adenocarcinoma. J Gastrointest Surg. (2012) 16:1478-86. doi: 10.1007/s11605-012-1923-5

15. Hamaguchi Y, Kaido T, Okumura S, Fujimoto Y, Ogawa K, Mori A, et al. Impact of quality as well as quantity of skeletal muscle on outcomes after liver transplantation. Liver Transpl. (2014) 20:1413-9. doi: 10.1002/lt. 23970

16. Liu X, Liu L, Chang K, Ye D, Zheng Y, Yao X. Risk factors of perioperative complications in patients undergoing radical retropubic prostatectomy: a ten-year experience. J Huazhong Univ Sci Technol. (2017) 37:37983. doi: 10.1007/s11596-017-1743-7

17. Psutka SP, Carrasco A, Schmit GD, Moynagh MR, Boorjian SA, Frank I, et al. Sarcopenia in patients with bladder cancer undergoing radical cystectomy impact on cancer-specific and all-cause mortality. Cancer. (2014) 120:29108. doi: $10.1002 / \mathrm{cncr} .28798$

18. Shen W, Punyanitya M, Wang Z, Gallagher D, St.-Onge MP, Albu J, et al. Total body skeletal muscle and adipose tissue volumes: estimation from a single abdominal cross-sectional image. J Appl Physiol. (2004) 97:23338. doi: 10.1152/japplphysiol.00744.2004 
19. Tan BH, Birdsell LA, Martin L, Baracos VE, Fearon KC. Sarcopenia in an overweight or obese patient is an adverse prognostic factor in pancreatic cancer. Clin Cancer Res. (2009) 15:6973-9. doi: 10.1158/1078-0432.CCR-09-1525

20. Wan F, Zhu Y, Gu C, Yao X, Shen Y, Dai B, et al. Lower skeletal muscle index and early complications in patients undergoing radical. World J Surg Oncol. (2014) 12:14. doi: 10.1186/1477-7819-12-14

21. Rutten IJ, Ubachs J, Kruitwagen RF, Van Dijk D, Beets-Tan R, Massuger L, et al. The influence of sarcopenia on survival and surgical complications in ovarian cancer patients undergoing primary debulking surgery. Eur J Surg Oncol. (2017) 43:717-24. doi: 10.1016/j.ejso.2016.12.016

22. Mason RJ, Boorjian SA, Bhindi B, Rangel L, Frank I, Karnes RJ, et al. The association between sarcopenia and oncologic outcomes after radical prostatectomy. Clin Genitourin Cancer. (2018) 16:e62936. doi: 10.1016/j.clgc.2017.11.003

23. McDonald A, Swain T, Mayhew D, Cardan R, Baker C, Harris D, et al. CT measures of bone mineral density and muscle mass can be used to predict noncancer death in men with prostate cancer. Radiology. (2017) 282:47583. doi: 10.1148/radiol.2016160626

24. Chang AJ, Autio KA, Roach M III, Scher HI. High-risk prostate cancer-classification and therapy. Nat Rev Clin Oncol. (2014) 11:30823. doi: $10.1038 /$ nrclinonc.2014.68

25. Martin L, Birdsell L, MacDonald N, Reiman T, Clandinin MT, McCargar LJ, et al. Cancer cachexia in the age of obesity: skeletal muscle depletion is a powerful prognostic factor, independent of body mass index. J Clin Oncol. (2013) 31:1539-47. doi: 10.1200/JCO.2012.45.2722

26. Ethgen O, Beaudart C, Buckinx F, Bruyere O, Reginster JY. The future prevalence of sarcopenia in Europe: a claim for public health action. Calcif Tissue Int. (2017) 100:229-34. doi: 10.1007/s00223-0160220-9
27. Herman MP, Raman JD, Dong S, Samadi D, Scherr DS. Increasing body mass index negatively impactsoutcomes following robotic radical prostatectomy. $J$ Soc Laparoendosc Surg. (2007) 11:438-42.

28. Calle EE, Rodriguez C, Walker-Thurmond K, Thun M. Overweight, obesity, and mortality from cancer in a prospectively studied cohort of U.S. adults. $N$ Engl J Med. (2003) 348:1625-38. doi: 10.1056/NEJMoa021423

29. Freedland SJ, Terris MK, Presti JCJ, Amling CL, Kane CJ, Trock B, et al. Obesity and biochemical outcome following radical prostatectomy for organ confined disease with negative surgical margins. J Urol. (2004) 172:5204. doi: 10.1097/01.ju.0000135302.58378.ae

30. Ruszat R, Bachmann A, Wyler S, Forster T, Zimmermann M, Sulser T. Einfluss des body-mass-index (BMI) auf die perioperativen Ergebnisse der endoskopischen radikalen Prostatektomie. Aktuelle Urol. (2006) 37:122. doi: 10.1055/s-2006-947511

31. Ha YS, Kim SW, Kwon TG, Chung SK, Yoo ES. Decrease in skeletal muscle index 1 year after radical cystectomy as a prognostic indicator in patients with urothelial bladder cancer. Int Braz J Urol. (2019) 45:68694. doi: 10.1590/s1677-5538.ibju.2018.0530

Conflict of Interest: The authors declare that the research was conducted in the absence of any commercial or financial relationships that could be construed as a potential conflict of interest.

Copyright (C) 2021 Angerer, Salomon, Beyersdorf, Fisch, Graefen and Rosenbaum. This is an open-access article distributed under the terms of the Creative Commons Attribution License (CC BY). The use, distribution or reproduction in other forums is permitted, provided the original author(s) and the copyright owner(s) are credited and that the original publication in this journal is cited, in accordance with accepted academic practice. No use, distribution or reproduction is permitted which does not comply with these terms. 